\title{
Apathy and Leukoaraiosis in Mild Cognitive Impairment and Alzheimer's Disease: Multicenter Diagnostic Criteria according to the Latest Studies
}

\author{
Carmen María Sarabia-Cobo ${ }^{a} \quad$ Victoria Pérez $^{b} \quad$ Carmen Hermosilla $^{b}$ \\ María José Nuñez ${ }^{b}$ Pablo de Lorenab

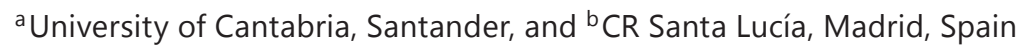

Key Words

Apathy · Mild cognitive impairment · Alzheimer's disease · Neuroimaging · Predictors .

White matter

\section{Abstract}

Aims: The aim is to study the prevalence and possible relationship of apathy and leukoaraiosis in cases of cognitive impairment of varying severity in Spain. Methods: We conducted a cross-sectional, descriptive, multicenter study involving 109 patients with Alzheimer's disease (AD) and 59 with mild cognitive impairment (MCI). Results: The older group with AD had a higher prevalence of leukoaraiosis and apathy, with significant differences compared to the MCI group. Conclusions: To our knowledge, this is the first multicenter study in our country that jointly analyzes the presence of apathy and leukoaraiosis in the institutionalized elderly with varying degrees of cognitive impairment according to the most recent criteria for detecting apathy in dementia.

(C) 2014 S. Karger AG, Basel

\section{Introduction}

Alzheimer's disease (AD) is the leading cause of dementia and is characterized by a progressive decline of cognitive function, which typically begins with memory impairment. Ferri et al. [1], in a Delphi study published in 2005, estimated that 24 million individuals have dementia, a figure that is predicted to double every 20 years until 2040 with a forecast of 81 million patients in 2005. The last global report on AD published in 2012 [2], following the estimates in the 2010 report [3], indicates that in 2030 there will be 66 million AD patients, 
increasing to 115 million in 2050. The WHO data published in 2012 are even more alarming: a new case of $\mathrm{AD}$ is diagnosed every $4 \mathrm{~s}$, with an estimated 682 million subjects suffering from dementia over the next 40 years unless an effective treatment or cure [4] is found.

The latest recommendations by the National Institute of Neurological Disorders and Communication and the Association of Related Alzheimer's Disease in the United States (NINCDS-ADRDA) for the diagnosis of AD disorders [5] and mild cognitive impairment (MCI) [6] emphasize the importance of early detection, broadening diagnostic criteria to items such as biomarkers, neuroimaging of specific injuries, and a battery of psychological tests that is as complete as possible.

Given the fact that MCI is a taxonomy that has become particularly relevant in recent years and based on the ever-growing literature on the subject that speaks of a high percentage of patients progressing to $\mathrm{AD}[7,8]$, it is interesting to look for factors that may influence the evolution towards dementia in order to establish early screening and therapeutic measures. This is not only due to the repercussions of detecting MCI cases but also to the fact that, regarding diagnosis, there still do not exist diagnostic instruments that are based on the recent recommendations $[9,10]$.

The presence of leukoaraiosis and the high prevalence of apathy have been addressed together in very few studies. This is why the two concepts that have gained prominence in recent years in relation to dementia will be addressed in this study. Our research is a pioneer study in our country as it addresses the prevalence of both processes in patients with MCI and AD according to recent consensus criteria that have been developed for the diagnosis of apathy, $\mathrm{MCI}$, and $\mathrm{AD}$. The term radiological leukoaraiosis is a nonspecific term found in computed tomography (CT) or magnetic resonance imaging (MRI) and denotes patches of hypo- or hyperintensity mainly in periventricular white matter and subcortical regions [11]. These ischemic lesions originate from an affectation of small vascular vessels and, hence, are also known as nonspecific white matter changes and small vessel ischemia. These lesions are frequently detected in elderly patients, with a higher prevalence in individuals with cerebrovascular disease, hypertension or diabetes, which are known to act as risk factors in the elderly [12-14]. Clinical manifestations may be absent; however, they are sometimes present with different cognitive affectations [15]. The prevalence is unclear because there is no consensus in the literature reviewed; the ranges found vary from 5 to 95\% [16]. Apathy and leukoaraiosis are mostly detected in asymptomatic individuals over 60 and in subjects with cognitive impairment, particularly in cases of cerebrovascular disease or vascular risk factors for ischemic stroke. Among patients with suspected cognitive impairment of vascular origin, leukoaraiosis is detected by CT between 41 and 100\% [15, 17] and between 64 and 100\% by MRI. AD ranges from 19 to $78 \%$ in studies of CT and from 7.5 to $100 \%$ in studies of brain MRI, but usually the changes in the white matter are less severe than those in patients with cerebrovascular disease [18]. Leukoaraiosis in the healthy population is less intense and less frequent than in patients with dementia [19]; it is usually detected in $21 \%$ by CT and in $100 \%$ of those who are evaluated with MRI. Leukoaraiosis is therefore a finding of heterogeneous neuroimaging that may be associated with various clinical and pathological situations with a range of very wide prevalence, since there is not yet a clear consensus in the scientific community for its correct identification. To date, there is evidence of a significant relationship between leukoaraiosis and impaired cognitive functions (language, attention, memory, etc.) [18], but there are few studies linking leukoaraiosis and dementia. Moreover, other important processes in the lives of patients such as psycho-emotional and/or psychiatric processes are also altered in dementia [20]. Of the possible emotional affective disorders that can present with dementia, the most prevalent is apathy.

The term apathy is frequently used in the neuropsychiatric literature, but it is not included as a separate term in the current nosological classifications such as the DSM-IV [21] and ICD-10 [22]. Apathy can be defined as a persistent deficit of motivation, a lack or decrease in 
feelings, emotions, or interests that give rise to a significant reduction in self-generated goaldirected behavior $[23,24]$. Apathy can be diagnosed with several standardized scales. However, they are not unified so that the percentage of AD patients suffering from apathy varies markedly between 19 and $76 \%[25,26]$. However, apathy is the most frequent symptom in neuropsychiatric $\mathrm{AD}[27,28]$ and other dementias, and is associated with increased cognitive and functional impairment and a high burden on the caregiver $[29,30]$. There is also a growing body of evidence that apathy is a prominent feature of predementia states [31-33]. In fact, there are indications that apathy could be a predictor of progression to dementia [34] to the point that the severity of apathy would be closely related to cognitive function [35]. Robert et al. [36] evaluated patients meeting the criteria of amnestic MCI using the Apathy Inventory (AI) and then checked whether AD had evolved after 1 year. The results showed that the cognitive function of patients with MCI who had apathy was higher than that of those in whom apathy was not present. However, given its high prevalence in dementia cases in almost all studies, a study from 2008 developed a set of several international associations to establish a consensus on the diagnostic criteria for apathy, mainly in AD cases [33].

Following the new consensus on leukoaraiosis and apathy, especially in cases of dementia, this study was proposed with the intent of establishing the prevalence and possible association of leukoaraiosis and apathy in two clinical groups, one with MCI (prodromal state as possible $\mathrm{AD}$ ) and another with $\mathrm{AD}$ institutionalized in seven residential centers in Spain. To our knowledge, it is the first study of its kind serving the two above factors (leukoaraiosis and apathy) conducted in Spain, using the new diagnostic criteria for apathy.

\section{Methods}

The present work is a cross-sectional, descriptive, multicenter study. The sample consisted of 109 patients diagnosed with probable AD and 59 diagnosed with $\mathrm{MCI}$ according to the latest criteria of the NINCDS-ADRDA [5, 6] published at the time of data collection by the relevant departments of neurology. All patients were residents in seven senior centers (belonging to the same foundation) located in seven cities in Spain, were aged 65 years or older and of both sexes. The subjects with $\mathrm{AD}$ at evolutionary stages 5 and 6 of the Global Deterioration Scale and Functional Assessment Staging (GDS and FAST, respectively) and MCI were selected, and all were diagnosed or reviewed by a neurologist within 6 months prior to the study. Reasons for exclusion were present sensory deficits that would prevent them from responding to tests, being diagnosed with depression by a psychiatrist (diagnosed by the Cornell Scale for Depression in Dementia [37]), and/or taking antidepressant treatment. Sociodemographic variables of age, sex, years of schooling, most prevalent chronic diseases, and scores on the following instruments were collected: (i) for the evaluation of apathy, the NPI-apathy [38] subscale was used. It is one of the most widely used scales and follows the consensus criteria established in 2008 [33]. It is part of a larger questionnaire, the Neuropsychiatric Inventory (NPI), which to date has proven to be the most valid and reliable assessment of neuropsychiatric symptoms in subjects with dementia. It was initially developed by Cummings et al. [39] to evaluate and quantify neurobehavioral disturbances in patients with dementia and to quantify the overhead caused by the caregiver situation. The score ranges from 0 to 12 , with higher scores revealing more severe apathy, and sets the cutoff at 4. For this study, a Spanish adaptation of the NPI scale [40] was used. (ii) For the diagnosis of leukoaraiosis, MRIs were performed with sounding devices of the same type (both hospitals gave their consent to the evaluation of the images for the study, as did the private schools where the tests were performed). The results were interpreted by two blinded neuroradiologists as to diagnosis of the patient and the study itself. They followed the directions on the Fazekas scale [41], which 
Table 1. Averages and percentages of sociodemographic variables and other tests

\begin{tabular}{l|l}
\hline \multicolumn{2}{l}{ Dement Geriatr Cogn Disord Extra 2014;4:228-235 } \\
\hline DOI: $10.1159 / 000363227$ & $\begin{array}{l}\text { C 2014 S. Karger AG, Basel } \\
\text { www.karger.com/dee }\end{array}$ \\
\hline
\end{tabular}

Sarabia-Cobo et al.: Apathy and Leukoaraiosis in Mild Cognitive Impairment and Alzheimer's Disease: Multicenter Diagnostic Criteria according to the Latest Studies

\begin{tabular}{lccl}
\hline & $\begin{array}{l}\text { MCI } \\
(\mathrm{n}=59)\end{array}$ & $\begin{array}{l}\text { AD } \\
(\mathrm{n}=109)\end{array}$ & $\mathrm{p}$ \\
\hline Age, years & $77.8(5.47)$ & $82.3(5.66)$ & 0.124 \\
Female, \% & 66 & 73 & 0.09 \\
Years of schooling & $6.24(3.75)$ & $6.08(2.40)$ & 0.165 \\
Chronic diseases, \% & & & \\
$\quad$ Hypertension & 63.8 & 69 & 0.02 \\
$\quad$ Diabetes mellitus & 31.7 & 29.6 & 0.321 \\
$\quad$ Hyperlipidemia & 26.8 & 35.4 & 0.078 \\
MEC & $28.05(0.93)$ & $19.6(2.17)$ & 0.01 \\
Barthel & $75(0.90)$ & $55(1.63)$ & 0.00 \\
\hline
\end{tabular}

Values are represented as mean (SD), unless otherwise indicated.

The $\mathrm{p}$ values were determined either by Student's $t$ (quantitative variables) or the $\chi^{2}$ (qualitative variables) tests; $\mathrm{p}<0.05$.

is one of the most frequently used scales. It is classified as grade 0 , absence of injury; grade 1 , the existence of focal lesions; grade 2, at the beginning of the confluence of injuries, and grade 3 , diffuse lesions comprising whole regions [18]. (iii) The Barthel test was administered to assess dependence. (iv) The participants were submitted to the Mini-Mental State Examination test using the Wolf method (MEC) to assess cognitive status. Data collection was carried out during 2012 and 2013. The sociodemographic variables were taken from the medical records and the apathy scale. The Barthel test and MEC were collected by the same researcher in collaboration with the medical and nursing staff of the respective residences. Leukoaraiosis diagnosis was made by two neuroradiologists within \pm 3 months after the collection of the data. Consent was obtained from the general management of the foundation, the residential centers, the ethics committee of the main residential center (in Madrid), and from all patients and/or guardians who were properly informed. The statistical treatment of the data was performed with SPSS 19.0 software. The researchers conducted a descriptive and a frequency analysis depending on each variable. The Kolmogorov-Smirnov (KS) test was used to examine normality, and the Levene test was used to evaluate the homogeneity of variances. If the criteria were met (normality and homoscedasticity), a parametric test was used: Student's t test (for quantitative variables) and the $\chi^{2}$ test (for qualitative variables) to compare the two groups. Correlation tests were also used to establish a possible link between quantitative (Pearson) and semiquantitative (Spearman) parameters, as appropriate ( $\alpha=0.05$ in all cases).

\section{Results}

Regarding sociodemographic variables and other collections, of the 109 subjects with $\mathrm{AD}, 74 \%$ were female, had a mean age of 82.3 years, $57.1 \%$ were at stage 5 , and $42.9 \%$ were at stage 6 GDS. Regarding the 59 patients with MCI, $64.3 \%$ were women, had a mean age of 77.8 years, and $67.8 \%$ were amnestic MCI. The KS test indicated normality (KS $=0.083, \mathrm{p}=$ 0.200 ), and Levene homoscedasticity $(17.27, \mathrm{p}=0.190)$ was also indicated. Parametric tests were conducted to evaluate the potential significant differences between the groups. The researchers conducted a MANOVA analysis to analyze the presence of possible differences in the scores of all parameters depending on the residence center and found no significant differences between the centers $[F(1,98)=0.879, p>0.05]$. 
Dementia

and Geriatric

Table 2. Percentages of leukoaraiosis and apathy as well as statistical significance
Sarabia-Cobo et al.: Apathy and Leukoaraiosis in Mild Cognitive Impairment and Alzheimer's Disease: Multicenter Diagnostic Criteria according to the Latest Studies

\begin{tabular}{llll}
\hline & MCI & AD & $p$ \\
\hline Leukoaraiosis & $46.8 \%$ & $67.9 \%$ & 0.00 \\
Apathy & $37.6 \%$ & $71.4 \%$ & 0.00 \\
\hline
\end{tabular}

The $\mathrm{p}$ values were determined by the $\chi^{2}$ test. $\mathrm{p}<0.05$.

Fig. 1. Percentages of leukoaraiosis and apathy in both groups.

Fig. 2. Percentages of chronic disease in patients with leukoaraiosis.
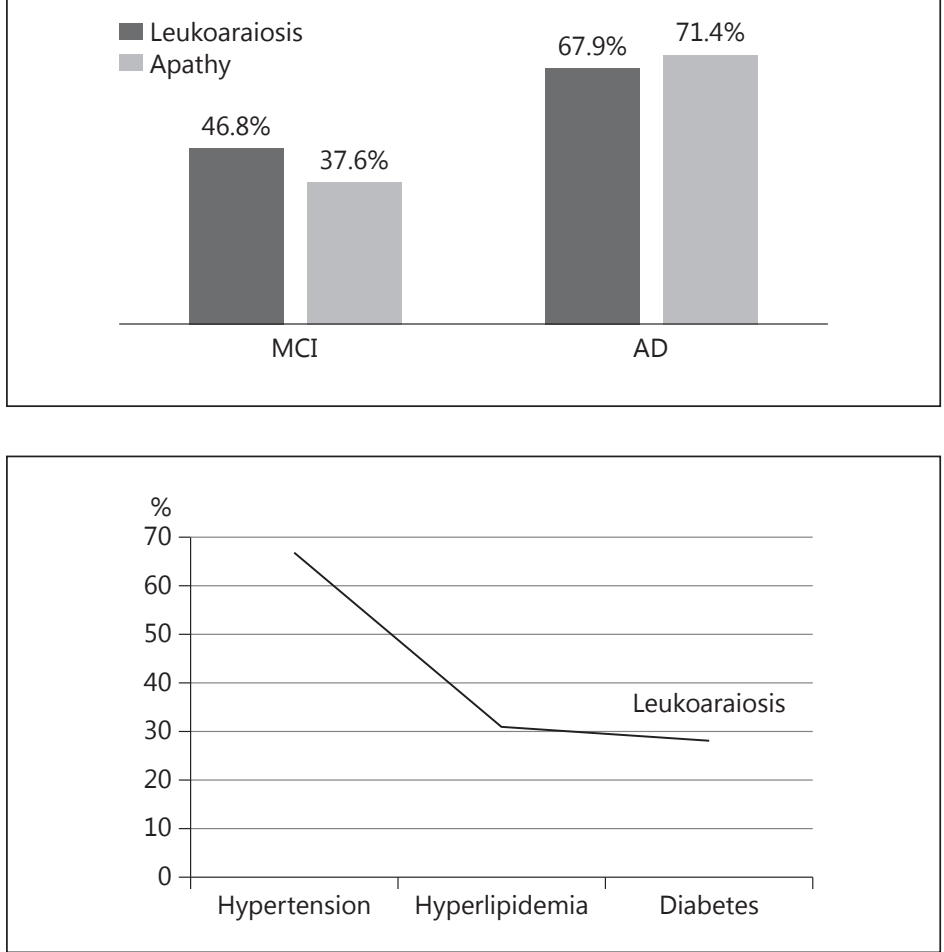

Table 1 shows the distribution of the groups by sociodemographic variables, background, Barthel scores, and MEC, along with the statistical significance tests performed (Student's t test for quantitative variables and the $\chi^{2}$ test for qualitative variables). It was found that there were no significant differences between the groups for age, sex, years of schooling as well as for diabetes and hyperlipidemia. There were significant differences in hypertension, MEC, and Barthel scores, resulting in the $\mathrm{AD}$ group being dependent on assistance and exhibiting a greater degree of cognitive impairment.

Regarding the presence of leukoaraiosis and apathy, the results for both groups are presented in figure 1 and table 2. They are coded in the presence/absence of diagnosis, with the significance test revealing significant differences between the groups, with greater presence of both apathy and leukoaraiosis in the older group with AD.

On the other hand, in relation to chronic diseases present, as can be seen in figure 2, patients with both $\mathrm{MCI}$ and $\mathrm{AD}$ who had hypertension were those with the higher percentages of leukoaraiosis (67\%) compared to other diseases such as dyslipidemia (31\% with leukoaraiosis) and diabetes (28\%). Upon performing a test for statistical significance between those with and those without leukoaraiosis $(n=168)$, statistically significant differences were only shown for hypertension $\left(\chi^{2}=0.874, p=0.03\right)$. 
Moreover, a correlation study between the different parameters was carried out. The existence of a positive correlation between age and leukoaraiosis ( $\mathrm{rs}=0.567, \mathrm{p}=0.00$ ) but not between age and apathy ( $\mathrm{rs}=0.236, \mathrm{p}=0.085$ ) was observed. A correlation between apathy and leukoaraiosis ( $r s=0.478, p=0.01$ ) was also found. In addition, the results of the correlation between the stage of EA and apathy were significant ( $\mathrm{rs}=0.568, \mathrm{p}=0.00$ ), as were those between AD and the stage of leukoaraiosis ( $r s=0.654, \mathrm{p}=0.00$ ). Regarding the presence of leukoaraiosis and MEC ( $\mathrm{rs}=0.685, \mathrm{p}<0.05$ ), a negative correlation between apathy and MEC ( $r s=0.425, \mathrm{p}<0.05$ ) was also found.

\section{Discussion}

The aim of this work was to study the prevalence of and possible relationship between apathy and leukoaraiosis in two groups of patients with different degrees of cognitive impairment (MCI and AD). Apathy is the most prevalent neuropsychiatric symptom in dementia, and leukoaraiosis is a frequent finding in cases of cognitive impairment. Our results showed a high prevalence of leukoaraiosis and apathy in AD patients and to a lesser but still significant extent in patients with MCI. Apathy and leukoaraiosis negatively correlated with patients' scores using MEC, indicating greater cognitive apathy and increased presence of leukoaraiosis impairment in these patients. Leukoaraiosis showed a positive correlation with age, occurring to a greater degree in older patients. Furthermore, a significant relationship was found between the presence of apathy and leukoaraiosis in both $\mathrm{MCI}$ and $\mathrm{AD}$, a finding that has been little studied [20] and that may open new avenues of research linking the presence of both as a prognostic factor for the evolution and severity of dementia. We also found a statistical significance between the presence of leukoaraiosis and hypertension in patients, a finding supported by the literature, with various research linking leukoaraiosis with cardiovascular disease $[12,14,19]$ as leukoaraiosis is associated with a reduction in blood perfusion in areas where it appears [42]. These results are consistent with the findings of other studies that found a high prevalence of apathy and leukoaraiosis in patients with dementia. Examples are the recent work by Mulin et al. [43] who found apathy in 55\% of the patients with AD as well as the one by Starkstein et al. [26] who suggested that apathy in AD is a marker of evolution characterized by a more rapid progression of cognitive, functional, and emotional impairment compared to patients without apathy. This would be an issue for future research, to assess whether patients presenting with MCI progress to AD apathy as well as for how long and how severe, to propose possible regression studies. As for the strong presence of leukoaraiosis and its association with impaired cognitive function, these results are consistent with other research that indicates a positive significant relationship with cognitive impairment: a greater presence of leukoaraiosis leads to a greater cognitive deterioration $[14,18,44,45]$. We also found a significant relationship between age and leukoaraiosis, especially in older patients who have a higher prevalence of leukoaraiosis, as confirmed in previous studies $[16-20,46]$. In the case of the MCI group that is currently treated as prodromal AD and in which the concept of Dubois' ADProd [47] is used, it seems clear that the significant presence of apathy and leukoaraiosis should be assessed and investigated in more detail within the framework predictors that indicate progression to AD [46] with all the remarkable consequences of an early screening and deterioration approach [48]. Our recent study has used consensus criteria established in the scientific literature regarding apathy. Although a consensus also needs to be established for leukoaraiosis, our results are consistent: there is a clear relationship between these symptoms and the presence of dementia, both increasing as the disease progresses. In addition to age and the presence of hypertension as factors confirming the presence of leukoaraiosis, the study was a very interesting method of 
research, especially in cases of vascular dementia and frontotemporal dementia $[19,49]$. As far as we know, few studies have jointly analyzed the presence of apathy and leukoaraiosis. The presence of both in AD patients is reported in the literature, but the heterogeneity of results is a clear indication of the need to continue reaching consensus diagnostic criteria. In conclusion, the presence of apathy and leukoaraiosis is associated with cognitive impairment and may prove to be a predictor of future dementia through the study of populations with $\mathrm{MCI}$. The growing aging population, which is associated with a higher prevalence of leukoaraiosis and chronic diseases such as hypertension, coupled with advances in neuroimaging techniques and the establishment of new criteria for detecting apathy establishes a scenario that advocates the early detection of dementia to establish preventive treatments that halt its advance in the general population.

\section{References}

$>1$ Ferri CP, Prince M, Brayne C: Global prevalence of dementia: a Delphi consensus study. Lancet 2005;366: 2112-2117.

2 Batsch NL, Mittelman MS: World Alzheimer Report 2012: Overcoming the Stigma of Dementia. London, Alzheimer's Disease International, 2012.

3 Wimo A, Prince M: World Alzheimer Report 2010: The Global Economic Impact of Dementia. London, Alzheimer's Disease International, 2010.

4 World Health Organization: Dementia: A Public Health Priority. Geneva, World Health Organization, 2012.

5 McKhann GM, Knopman DS, Chertkow H, Hyman BT, Jack CR Jr, Kawas CH, et al: The diagnosis of dementia due to Alzheimer's disease: recommendations from the National Institute on Aging-Alzheimer's Association workgroups on diagnostic guidelines for Alzheimer's disease. Alzheimers Dement 2011;7:263-269.

-6 Albert MS, DeKosky ST, Dickson D, Dubois B, Feldman HH, Fox NC, et al: The diagnosis of mild cognitive impairment due to Alzheimer's disease: recommendations from the National Institute on Aging-Alzheimer's Association workgroups on diagnostic guidelines for Alzheimer's disease. Alzheimers Dement 2011;7:270279.

7 Petersen RC, Roberts RO, Knopman DS, Boeve BF, Geda YE, Ivnik RJ, et al: Mild cognitive impairment: ten years later. Arch Neurol 2009;66:1447-1455.

-8 Sánchez-Rodríguez JL, Torrellas-Morales C: A review of the construct of mild cognitive impairment: general aspects (in Spanish). Rev Neurol 2011;52:300-305.

-9 Mora-Simón S, García-García R, Perea-Bartolomé MV, Ladera-Fernández V, Unzueta-Arce J, Patino-Alonso MC, et al: Mild cognitive impairment: early detection and new perspectives (in Spanish). Rev Neurol 2012;54: 303-310.

10 Guo LH, Alexopoulos P, Eisele T, Wagenpfeil S, Kurz, A, Perneczky R: The National Institute on AgingAlzheimer's Association research criteria for mild cognitive impairment due to Alzheimer's disease: predicting the outcome. Eur Arch Psychiatry Clin Neurosci 2013;263:325-333.

11 Pantoni L, Garcia JH: Pathogenesis of leukoaraiosis: a review. Stroke 1997;28:652-659.

-12 Longstreth WT Jr, Arnold AM, Beauchamp NJ Jr, Manolio TA, Lefkowitz D, Jungreis C, et al: Incidence, manifestations, and predictors of worsening white matter on serial cranial magnetic resonance imaging in the elderly: the Cardiovascular Health Study. Stroke 2005;36:56-61.

13 Schwartz GL, Fornage M, Mosley T, Turner ST: Treatment of leukoaraiosis. Curr Treat Options Cardiovasc Med 2005; 7:173-177.

14 Srikanth V, Beare R, Blizzard L, et al: Cerebral white matter lesions, gait, and the risk of incident falls: a prospective population-based study. Stroke 2009;40:175-180.

15 Echávarri C, Caballero MC, Aramendía AI, Cabada T: Histological correlation study of leukoaraiosis on MRI studies in post-mortem brains with Alzheimer's disease and other dementias (in Spanish). Real Invest Demenc (Barcelona) 2010;44:15-23.

16 Bohnen NI, Müller ML, Zarzhevsky N, Koeppe RA, Bogan CW, Kilbourn MR, et al: Leucoaraiosis, nigrostriatal denervation and motor symptoms in Parkinson's disease. Brain 2011;134:2358-2365.

17 Ramírez SV, Álvarez E, Paradela C, Álvarez E: Leukoaraiosis. Pathophysiology and diagnostic imaging (in Spanish). Rev Hab Cien Med 2013;12:336-342.

18 Jiménez I, Agulla J, Pouso M, Sabucedo M, Rodríguez-Yánez M, et al: Cognitive impairment associated to leukoaraiosis: its pathophysiology, clinical manifestations and treatment (in Spanish). Rev Neurol 2008;47:536544.

19 Appelman AP, Vincken KL, Van der Graaf Y: White matter lesions and lacunar infarcts are independently and differently associated with brain atrophy: the SMART-MR study. Cerebrovasc Dis 2010;29:28-35.

20 Thomas P, Hazif-Thomas C, Saccardy F, Vandermarc P: Leukoaraiosis. Demotivation and frontal dysfunction in the elderly; in Salvà A, Vellas B (directores): Año gerontológico. Barcelona, Editorial Glosa, 2003, pp 193-207. 
21 Starkstein SE, Leentjens AF: The nosological position of apathy in clinical practice. J Neurol Neurosurg Psychiatry 2008;79:1088-1092.

22 Janca A, Ustun TB, Early TS, Sartorius N: The ICD-10 symptomchecklist: a companion to the ICD-10 classification of mental and behavioural disorders. Soc Psychiatry Psychiatr Epidemiol 1993;28:239-242.

23 Levy R, Dubois B: Apathy and the functional anatomy of the prefrontal cortex-basal ganglia circuits. Cereb Cortex 2006;16:916-928.

24 Agüera-Ortiz LF, Gil-Ruiz N, Cruz-Orduña I, Ramos-García MI, Osorio-Suárez RM, Valentí-Soler M: Development of a scale for the measurement of apathy in institutionalized patients with Alzheimer-type dementia: the APADEM-NH-66 scale extended version (in Spanish). Psicogeriatría 2010;2:207-219.

25 Clarke DE, van Reekum R, Simard M, Streiner DL, Conn D, Cohen T, et al: Apathy in dementia: clinical and sociodemographic correlates. J Neuropsychiatry Clin Neurosci 2008;20:337-347.

26 Starkstein SE, Jorge R, Mizrahi R, Robinson RG: A prospective longitudinal study of apathy in Alzheimer's disease. J Neurol Neurosurg Psychiatry 2006;77:8-11.

27 Mega MS, Cummings JL, Fiorello T: The spectrum of behavioral changes in Alzheimer's disease. Neurology 1996;46:130e5.

28 Robert P, Verhey F, Byrne EJ, Hurt C, De Deyn PP, Nobili F, et al: Grouping for behavioral and psychological symptoms in dementia: clinical and biological aspects. Consensus paper of the European Alzheimer disease consortium. Eur Psychiatry 2005;20:490e6.

29 Landes AM, Sperry SD, Strauss ME, Geldmacher DS: Apathy in Alzheimer's disease. J Am Geriatr Soc 2001;49: $1700 \mathrm{e} 7$.

30 Onyike CU, Sheppard JM, Tschanz JT, Norton MC, Green RC, Steinberg M, et al: Epidemiology of apathy in older adults: the Cache County study. Am J Geriatr Psychiatry 2007;15:365e75.

-31 Ready RE, Ott BR, Grace J, Cahn-Weiner DA: Apathy and executive dysfunction in mild cognitive impairment and Alzheimer disease. Am J Geriatr Psychiatry 2003;11:222e8.

-32 Boyle PA, Malloy PF: Treating apathy in Alzheimer's disease. Dement Geriatr Cogn Disord 2004;17:91-99.

33 Robert P, Onyike CU, Leentjens AFG, Dujardin K, Aalten P, Starkstein S, Byrne J: Proposed diagnostic criteria for apathy in Alzheimer's disease and other neuropsychiatric disorders. Eur Psychiatry 2009;24: 98-104.

-34 Feldman H, Scheltens P, Scarpini E, Hermann N, Mesenbrink P, Mancione L, et al: Behavioral symptoms in mild cognitive impairment. Neurology 2004;62:1199e201.

-35 Hwang TJ, Masterman DL, Ortiz F, Fairbanks LA, Cummings JL: Mild cognitive impairment is associated with characteristic neuropsychiatric symptoms. Alzheimer Dis Assoc Disord 2004;18:17e21.

-36 Robert PH, Berr C, Volteau M, Bertogliati C, Benoit M, Sarazin M, et al: Apathy in patients with mild cognitive impairment and the risk of developing dementia of Alzheimer's disease: a one-year follow-up study. Clin Neurol Neurosurg 2006;108:733-736.

-37 Alexopoulos GS, Abrams RC, Young RC, Shamoian CA: Cornell scale for depression in dementia. Biol Psychiatry 1988;23:271-84.

-38 Clarke DE, Ko JY, Kuhl EA, van Reekum R, Salvador R, Marin RS: Are the available apathy measures reliable and valid? A review of the psychometric evidence. J Psychosom Res 2011;70:73-97.

39 Cummings JL, Mega M, Gray K, Rosenberg-Thompson S, Carusi DA, Gornbein J: The Neuropsychiatric Inventory: comprehensive assessment of psychopathology in dementia. Neurology 1994;44:2308-2314.

-40 Vilalta-Franch J, Lozano-Gallego M, Hernandez-Ferrandiz M, Llinas-Regla J, Lopez-Pousa, S, Lopez OL: The Neuropsychiatric Inventory. Psychometric properties of its adaptation into Spanish (in Spanish). Rev Neurol 1998;29:15-19.

41 Fazekas F, Chawluk JB, Alavi A, Hurtig H, Zimmerman RA: MR signal abnormalities at 1.5 T in Alzheimer's dementia and normal aging. AJNR Am J Roentgenol 1987;149:351-356.

42 Oda K, Okubo Y, Ishida R, et al: Regional cerebral blood flow in depressed patients with white matter magnetic resonance hyperintensity. Biol Psychiatry 2003;53:150-156.

43 Mulin E, Leone E, Dujardin K, Delliaux M, Leentjens A, Nobili F, et al: Diagnostic criteria for apathy in clinical practice. Int J Geriatr Psychiatry 2011;26:158-165.

44 Gouw A, Seewann A, Vrenken H, Van der Flier WM, Rozemuller JM, Barkhof F, et al: Heterogeneity of white matter hyperintensities in Alzheimer's disease: post-mortem quantitative MRI and neuropathology. Brain 2008;16:1-13.

-45 Tirapu-Ustárroz J, Luna-Lario P, Hernáez-Goñi P, García-Suescun I: Relation between white matter and cognitive functions (in Spanish). Rev Neurol 2011;52:725-742.

-46 Bartrés-Faz D, Clemente IC, Junqué C: White matter changes and cognitive performance in aging (in Spanish). Rev Neurol 2001;33:347-353.

47 Dubois B: 'Prodromal Alzheimer's disease': a more useful concept than mild cognitive impairment? Curr Opin Neurol 2000;13:367-369.

48 Valls-Pedret C, Molinuevo JL, Rami L: Early diagnosis of Alzheimer's disease: the prodromal and preclinical phase (in Spanish). Rev Neurol 2010;51:471-480.

49 van Straaten EC, Fazekas F, Rostrup E, Scheltens P, Schmidt R, Pantoni L, et al; LADIS Group: Impact of white matter hyperintensities scoring method on correlations with clinical data: the LADIS study. Stroke 2006;37: 836-840. 